\title{
Microsized subsurface modification of mono-crystalline silicon via non-linear absorption
}

\author{
V. V. Parsi Sreenivas \\ pars@bias.de
}

\section{Bülters}

\section{R. B. Bergmann}

BIAS, Bremer Institut für angewandte Strahltechnik, Klagenfurter Straße 2, 28359 Bremen, Germany

BIAS, Bremer Institut für angewandte Strahltechnik, Klagenfurter Straße 2, 28359 Bremen, Germany

We introduce a novel method of optically inducing microsized subsurface structures using non-linear absorption of near infrared light in mono-crystalline silicon. We discuss the physical processes such as multi-photon absorption and self focussing in the material. The results presented in this paper demonstrate a new method of subsurface modifications in silicon and may open up novel avenues for optical devices embedded in silicon and optical process for the separation of wafers from their ingots.

[DOI: http://dx.doi.org/10.2971/jeos.2012.12035]

Keywords: Silicon, non-linear absorption, opto-mechanical processes, silicon photonics

\section{INTRODUCTION}

Silicon, being the backbone of the semiconductor industry, has always been under thorough investigation and a matter of research. Crystalline silicon has been electrically, optically and mechanically characterised extensively [1]-[3] over decades. The introduction of mechanical stresses in silicon through optical means is a vibrant field [4] as it promises high density system-on-chip devices and optical interconnects. Here, we present a novel method of creating subsurface structures as a cornerstone for embedded optical devices [5] and a novel approach of optically separating wafers from their ingots [6, 7]. Although the multiphoton absorption process has been previously used to induce modifications in many optically transparent materials [8], this process has been demonstrated for the first time in silicon. This is due to physical parameters of silicon such as the low thermal coefficient, infrared transparency, etc., that limit the choice of the specifications of the laser system to be used. These parameters and their limitations are discussed in Section 2.

We exploit the non-linear properties of silicon [9] to optically induce mechanical stresses within its bulk. For this purpose we use a near infra red [10] laser source and a high numerical aperture (N.A.) objective[11] to focus a laser beam into the bulk of silicon to concentrate high electromagnetic energy densities into a tiny volume $\left(\sim \mu \mathrm{m}^{3}\right)$. This induces a series of non-linear reactions $[12,13]$ such as self phase modulation (SPM), self focussing, multiple photon absorption (MPA), avalanche ionisation, etc. These are then followed by generation of an electron plasma cloud [8] that exerts mechanical stresses to the silicon lattice which eventually leads to the formation of defects in regions where the beam was focussed. By spatially controlling these defective regions, one can create optically detectable subsurface structures. Figure 1(a) schemati- cally represents our proposed optical subsurface modification mechanism based on the approaches presented in $[6,7]$.

\section{LIGHT AND MATTER INTERACTIONS}

In this section, we discuss physical parameters involved in the process and the required parameters of the laser system to be used.

Following is the well known description of optical absorption in dielectric media where the induced polarisation $P(t)$ due to an applied electric field $E(t)$ in an isotropic material, assuming an instantaneous response, is given by

$$
P(t)=\varepsilon_{0} \chi^{(1)} E(t)+\varepsilon_{0} \chi^{(2)} E^{2}(t)+\varepsilon_{0} \chi^{(3)} E^{3}(t)+\ldots,
$$

where $\chi$ is the electrical susceptibility of the material and defines the order of the interaction between $E$ and $P$, and $\varepsilon_{0}$ is the vacuum permittivity. At a wavelength of $1550 \mathrm{~nm}$, the linear absorption coefficient in silicon is as low as $\sim 10^{-8} \mathrm{~cm}^{-1}$ and hence, the first term of the equation is neglected. Silicon belongs to the $m 3 m$ point group and is a centrosymmetric [14] crystal with two sets of mirror planes, $\{100\}$ and $\{110\}$. The absence of the second-order susceptibility term is a characteristic feature of crystals belonging to the $m 3 m$ point group. Therefore, the third order susceptibility is the largest contributing factor to the electromagnetically induced polarisation in silicon atoms and thus,

$$
P(t) \simeq \varepsilon_{o} \chi^{(3)} E^{3}(t)
$$

By introducing the frequency components of the $E$ field, one can expand Eq. (1) and represent the polarisation $(P)$ in terms of its frequency components and associate with them the 
(a)

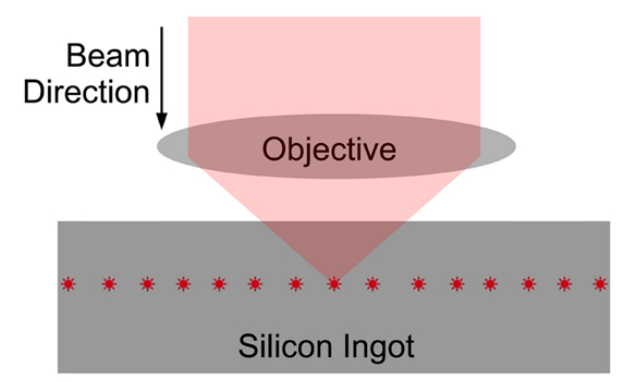

(b)

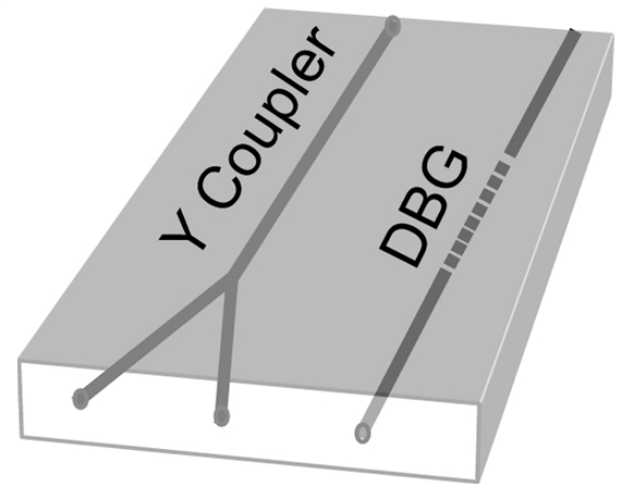

FIC. 1 (a) Schematic representation of the steps involved in the proposed subsurface modification process. When the position of the focus spot is moved over the entire area of the wafer, a pattern can be created within the volume of silicon. (b) The patterns are supposed to be used to design optical units in the bulk of silicon such as optical Y-couplers or Distributed Bragg Gratings (DBG) below the Si surface.

physical processes such as, SPM and third harmonic generation [9]. The SPM leads to a local increase in the focussed electromagnetic densities, which further initiates non-linear processes such as the MPA. The electromagnetic flux and the coefficients of absorption in MPA is governed by

$$
\begin{aligned}
-\frac{d}{d z} I(t, z) & =\alpha I(t, z)+\beta I^{2}(t, z)+\gamma I^{3}(t, z)+\ldots \\
& \simeq \beta I^{2}(t, z)+\gamma I^{3}(t, z)+\ldots,
\end{aligned}
$$

where $I$ is the intensity of the electromagnetic radiation, $z$ is the depth of absorption, $t$ the time variable and $\alpha, \beta, \gamma$ are the first, second and third order photon absorption coefficients, respectively. Since the absorption losses in silicon at $1550 \mathrm{~nm}$ are insignificant, the first term (linear) can be ignored. The value of $\beta$ is known to vary between 1.8 and $0 \mathrm{~cm} / \mathrm{GW}$ in a wavelength range between 800 and $2200 \mathrm{~nm}$ [15]. The maximum value in this range is $\sim 0.45 \mathrm{~cm} / \mathrm{GW}$ at $1550 \mathrm{~nm}$ [16], which is the wavelength of the laser used.

Figure 2 shows a schematic representation of the physical processes involved in the subsurface modification of silicon. Multi-photon absorption creates the initial (seed) electron density, which is then increased by the avalanche ionisation process. Initial ionisation takes place when a few seed electrons in the conduction band oscillate in the laser electromagnetic field and are accelerated to energies greater than the band gap of silicon. These high energy electrons then interact with the valence electrons and transfer enough energy to excite them to the conduction band. This iterative process

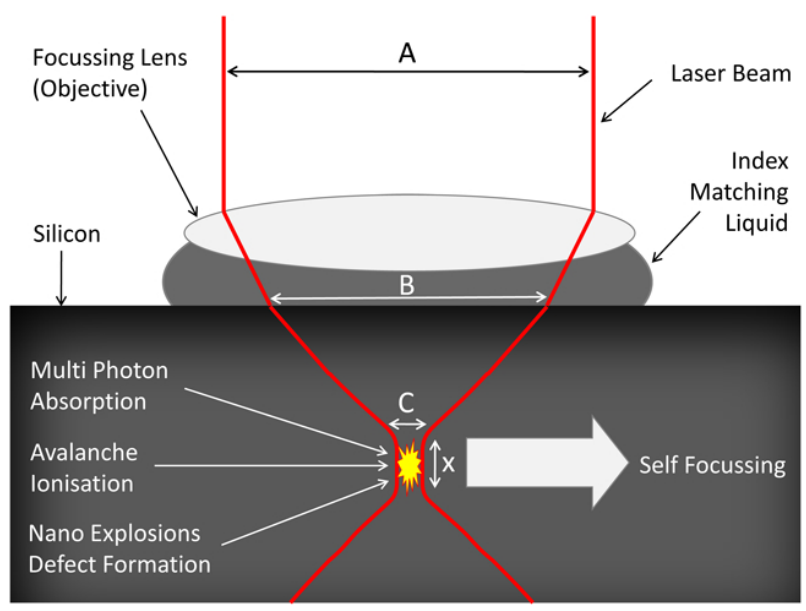

FIG. 2 Schematic representation of the physical process involved during the interaction of a focussed laser beam with silicon. ' $A$ ' is the initial beam waist, ' $B$ ' is the beam waist at the interface of index matching liquid a silicon and ' $C$ ' is the focussed spot diameter in the bulk of silicon. The local modification of the refractive index along the direction of beam propagation leads to the self focussing effect. This effect is spatially distributed over a region $x$, that is marked in this figure. Also, due to the high intensities, non linear processes such as MPA and avalanche ionisation occur that eventually lead to defect formation within the bulk of silicon. These effects are temporally distributed in the focussed region.

leads to an avalanche ionisation in the material. The deciding factor for the avalanche ionisation to be initiated by the multi-photon process is established by the Keldysh parameter $\left(\epsilon_{o s c} / \Delta_{g a p}\right)$ to be greater than unity. The parameters $\epsilon_{\text {osc }}$ and $\Delta_{\text {gap }}$ are the electron oscillation energy and the electronic band gap, respectively. The probability of multi-photon ionisation $P_{m p i}$ per atom per second, is given by

$$
P_{m p i} \approx \omega n_{p h}^{3 / 2}\left(\frac{\epsilon_{o s c}}{2 \Delta_{g a p}}\right),
$$

see ref. [17], where $n_{p h}$ is the number of photons involved in the transition of one electron and $\Delta_{g a p}=n_{p h} \hbar \omega$. Since the probability is directly proportional to the oscillation energy of the electron, the intensity of the incident electromagnetic radiation from the laser is critical in initiating the process. The subsequent generation of the electrons results in formation of an electron plasma cloud that exerts an outward pressure in the silicon crystal lattice. The damage caused by this pressure is largely dependent on the variation of the mechanical stress in different crystal orientations [18].

Based on the rate of photon absorption and electron-phonon interactions, the time span in which the MPA and the avalanche ionisation processes occur is calculated to be $10^{-14} \cdots 10^{-11}$ seconds [19]. The process of defect formation is dependent on the Young's modulus of the material and its thermal coefficients. For silicon, it is expected to be a couple of tens of nano seconds.

\section{EXPERIMENTAL RESULTS}

Based on the requirements described above, a laser system is used that has a centre wavelength of $1550 \mathrm{~nm}$, a pulse duration of $800 \mathrm{fs}$, a pulse energy of $50 \mu \mathrm{J}$ and a maximum repetition rate of $100 \mathrm{kHz}$. 
(a)

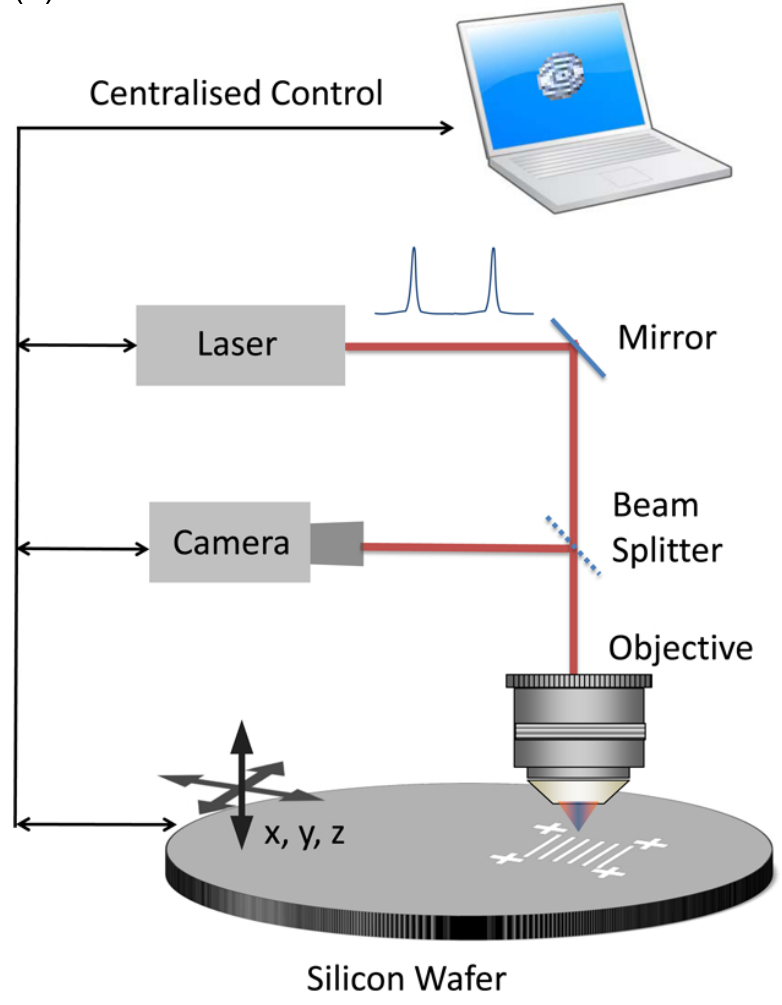

(b)

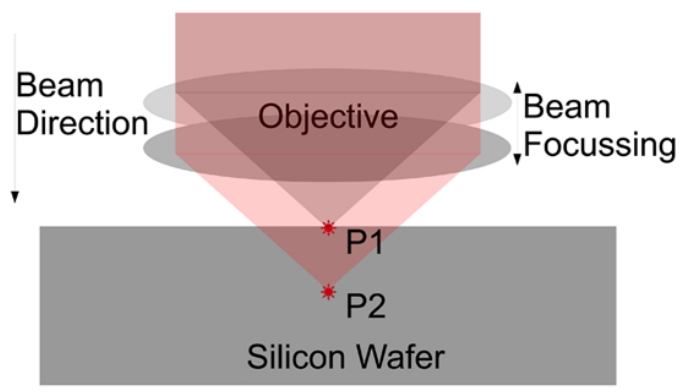

(c)

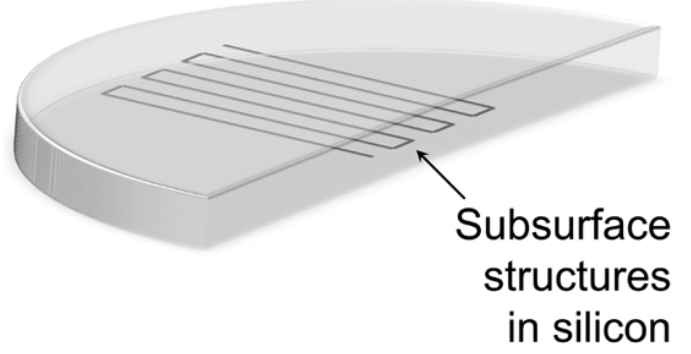

FIC. 3 (a) Experimental setup: The laser beam is focussed into the silicon wafer with an objective. The motion stages are programmed to drive the wafer into the laser focus spot and to move in a pre-defined pattern to create the desired structures. The camera is used to control the position of the focus. A silicon wafer with a thickness of $200 \mu \mathrm{m}$ is used so that it is easier to make the transmission light images after writing the structures as displayed in Figure 4 (b). (b) Schematic of the focussing mechanism of the setup shown in (a). $P_{1}$ and $P_{2}$ are the positions on the wafer and inside the wafer, respectively. As the beam is focussed into the wafer, structures are created at the depth of $P_{2}$ within the wafer. These are imaged using an IR microscope. (c) Schematic representation of the structures written in silicon.

Figure 3(a) shows the experimental setup. The laser beam is focussed using an oil immersion microscope objective with an N.A. of 1.25. Figure 3(b) shows the principal working area of

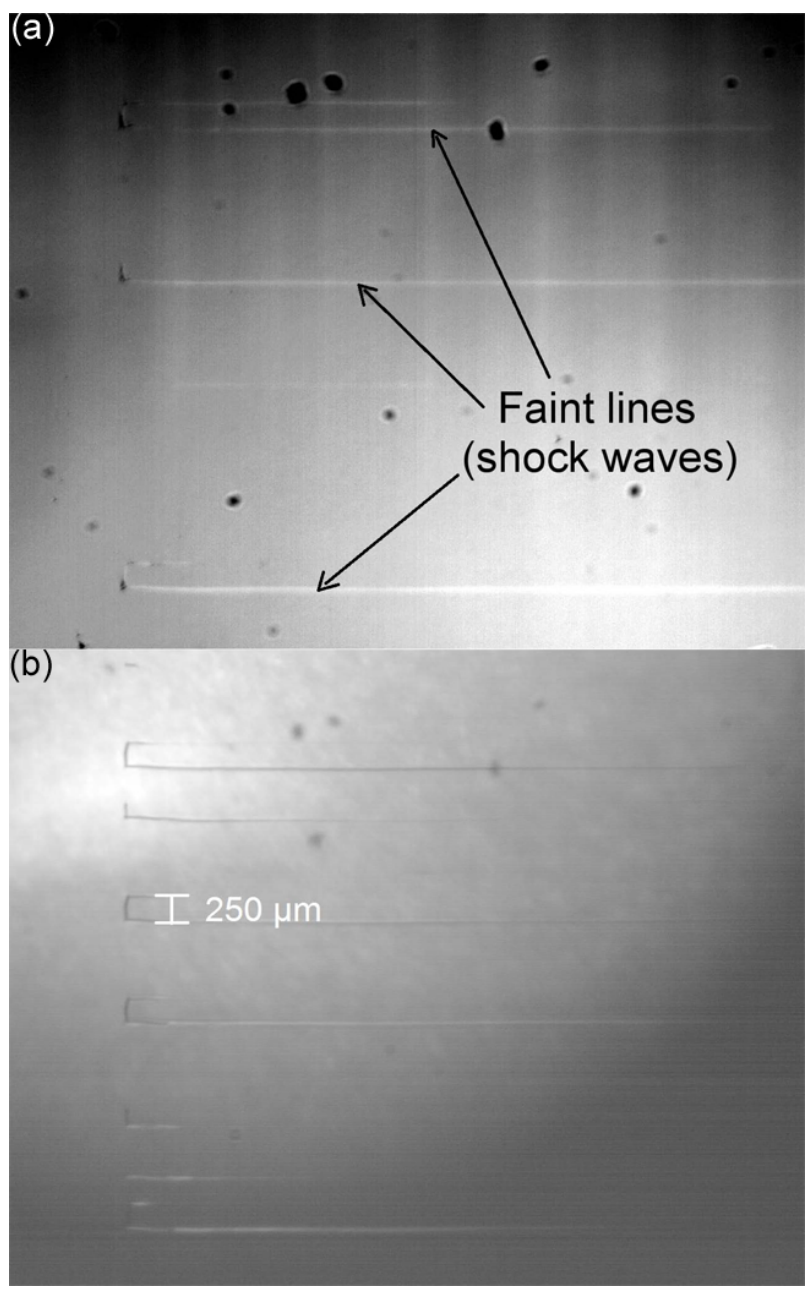

FIG. 4 (a) Contrast enhanced visible light image, taken from the surface facing the laser beam. A few faint lines can be seen which appear to be shock waves that have travelled from the bulk to the surface as described in [19]. (b) An infra red image of the same section reveals buried lines within the bulk of the silicon with a width of $\approx 5 \mu \mathrm{m}$. The neighbouring lines are $\approx 250 \mu \mathrm{m}$ apart.

the experiment where the focus of the laser beam is driven into the bulk of silicon to realise subsurface modifications. The position of the focus spot is currently monitored by the back reflection of the laser beam from silicon that is sampled into a camera. When positioned, the motion stages carrying the wafer is activated. Figure 3(c) schematically shows the subsurface pattern in a silicon wafer that is described throughout this paper.

Figure 4(a) and 4(b) show visible and infra red light images, respectively, of the structures written via this process. The two images reveal the buried structures in silicon with accompanying surface structures attributed to shock waves in some regions on the surface. The width of the buried structures as measured by a $40 \mathrm{x}$ objective is around $5 \mu \mathrm{m}$. For the writing process, the refractive index of the index matching liquid is 1.4917. The experiment was performed with a pulse energy of $50 \mu \mathrm{J}$. Also, as argued in the previous section and from [18], the directional dependency of the mechanical stresses in silicon was observed in our experiments. The wafer used was (100) oriented, highly doped silicon with a resistivity of 5 to 

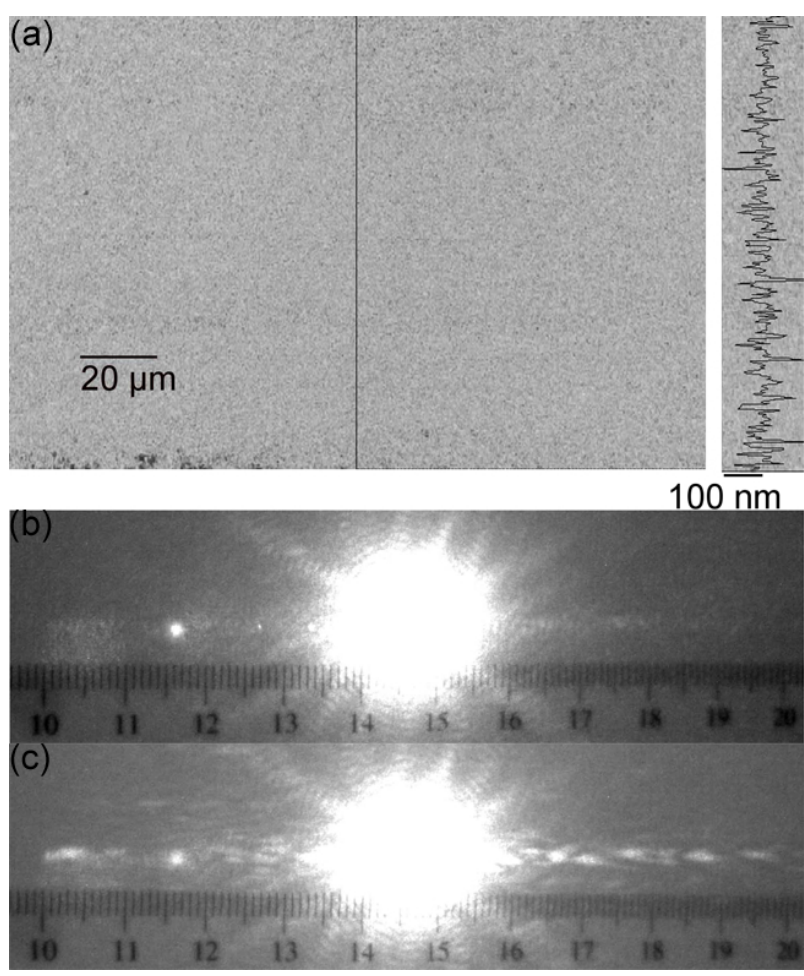

FIG. 5 (a) Silicon surface after subsurface modification. Right: Height profile as measured along the black line, close to the centre of the picture. The height variation is $\sim 200 \mathrm{~nm}$. (b) An image of laser light with a wavelength of $1064 \mathrm{~nm}$ passing through the unmodified single crystal silicon. The bright spot of the left side of the beam is an artefact of the imaging lens and must be ignored. (c) Diffraction pattern observed due to the subsurface modifications. These modifications arise from the diffraction grating, as show in Figure $3(\mathrm{c})$ with a spacing of $25 \mu \mathrm{m}$. A cm scale shows the dimensions of the pattern.

$50 \Omega \mathrm{cm}$ that showed traces of subsurface modification, also shown in Figure 4(b).

Figure 5 shows experiments performed with an optical grating with a line spacing of $25 \mu \mathrm{m}$. The structures are written using the procedure described in Figure 3. When these structures are observed under a visible light microscope, the surface shows irregularities as shown in Figure 5(a) presumably caused by shockwaves travelling to the surface. However, a surface scan reveals no periodicity in the roughness pattern. The height of the roughness is of the order of $200 \mathrm{~nm}$. The possible modifications of the material due to the processing described here is a subject of further investigation.

The structures were illuminated using a $1064 \mathrm{~nm}$ CW laser and a diffraction pattern is achieved which is projected at a distance of $56 \mathrm{~cm}$ from the wafer surface. Figure 5(b) shows the undiffracted light without the subsurface modifications, so as to visualise the differences from Figure 5(c) showing the diffracted light through the subsurface modifications. By ignoring an artefact of the imaging lens on the left hand side of the zeroth order, we use the right hand side of the pattern shown in Figure 5(c) to extract the intensities of the diffraction peaks as shown in Figure 6. Since the detailed geometry and the variation of the refractive index of the subsurface grating has not yet been determined, the position and shape of these peaks and the corresponding analytical model is a subject of further work.

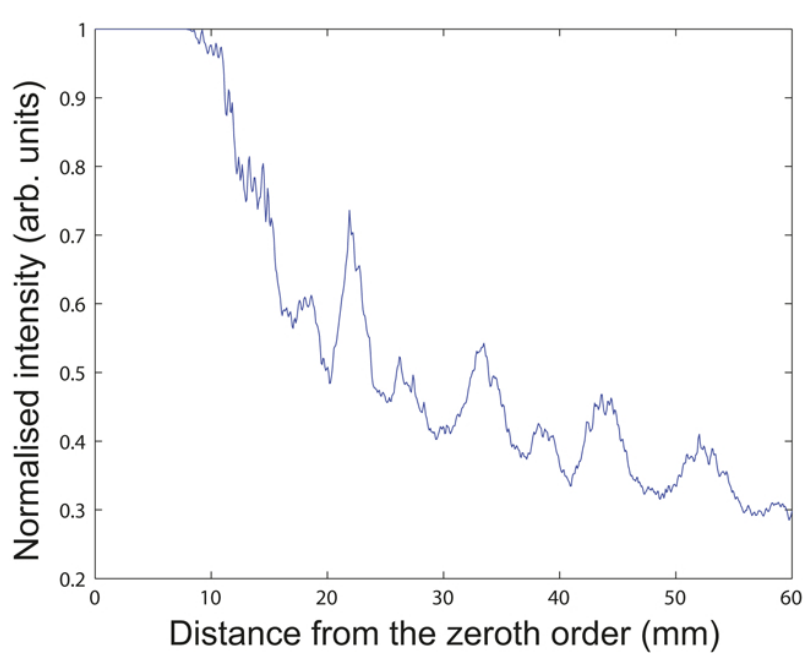

FIC. 6 Plot of the diffraction peak intensity against the distance of these peaks to the right from the central maxima, corresponding to Figure $5(\mathrm{c})$. The position and shape of the peaks is a subject of further investigation.

\section{CONCLUSIONS}

The formation of optically detectable buried structures in silicon is demonstrated as a new avenue to inscribe three dimensional structures for micro optical usage. Our approach may enable a technological breakthrough in terms of optical on-chip monolithic integration of optical devices. Further structural and optical characterisation as well as a thorough theoretical understanding and physical modelling of multiphoton absorption (MPA), self phase modulation (SPM) and self focussing processes in silicon are necessary.

\section{References}

[1] W. C. O'Mara, R. B. Herring, and L. P. Hunt, Handbook of Semiconductor Silicon Technology (first edition, Noyes Publications, New Jersey, 1990).

[2] R. B. Bergmann, Growth, Characterization and Electronic Applications of Si-based Thin Films (Research Signpost, Trivandrum, 2002).

[3] R. Bergmann, and J. Werner, "The future of crystalline silicon films on foreign substrates," Thin Solid Films 403, 162-169 (2002).

[4] A. H. Nejadmalayeri, P. R. Herman, J. Burghoff, M. Will, S. Nolte, and A. Tünnermann, "Inscription of optical waveguides in crystalline silicon by mid-infrared femtosecond laser pulses," Opt. Lett. 30, 964-966 (2005).

[5] R. Bergmann, M. Bülters, and V. V. Parsi Sreenivas, "Verfahren und Vorrichtung zum Herstellen von mindestens einem photonischen Bauelement," German Patent 102011113824.6 (2011).

[6] A. Hildebrand, and R. Bergmann, "Verfahren zum Separieren eines Halbleiter-Wafers von einem Halbleiterkristall," German Patent 10 2009005303.4 (2009).

[7] A. Hildebrand, and R. Bergmann, "Verfahren zum Separieren eines Halbleiter-Wafers von einem Halbleiterkristall," European Patent 10 150 890.1-2302 (2010).

[8] E. G. Gamaly, S. Juodkazis, K. Nishimura, H. Misawa, B. LutherDavies, L. Hallo, P. Nicolai, and V. T. Tikhonchuk, "Laser-matter interaction in the bulk of a transparent solid: Confined microexplosion and void formation," Phys. Rev. B 73, 214101 (2006). 
[9] J. Leuthold, C. Koos, and W. Freude, "Nonlinear silicon photonics," Nat. Photonics 4, 535-544 (2010).

[10] W. Spitzer, and H. Y. Fan, "Infrared Absorption in $n$-Type Silicon," Phys. Rev. 108, 268-271 (1957).

[11] J. B. Ashcom, R. R. Gattass, C. B. Schaffer, and E. Mazur, “Numerical aperture dependence of damage and supercontinuum generation from femtosecond laser pulses in bulk fused silica," J. Opt. Soc. Am. B 23, 2317-2322 (2006).

[12] M. C. Fischer, T. Ye, G. Yurtsever, A. Miller, M. Ciocca, W. Wagner, and W. S. Warren, "Two-photon absorption and self-phase modulation measurements with shaped femtosecond laser pulses," Opt. Lett. 30, 1551-1553 (2005).

[13] Q. Lin, 0. J. Painter, and G. P. Agrawal, "Nonlinear optical phenomena in silicon waveguides: modeling and applications," Opt. Express 15, 16604-16644 (2007).

[14] M. G. Kuzyk and C. W. Dirk, "Effects of centrosymmetry on the nonresonant electronic third-order nonlinear optical susceptibility," Phys. Rev. A 41, 5098-5109 (1990).
[15] A. D. Bristow, N. Rotenberg, and H. M. van Driel, "Two-photon absorption and Kerr coefficients of silicon for 850-2200 nm," Appl. Phys. Lett. 90, 191104 (2007).

[16] H. K. Tsang, C. S. Wong, T. K. Liang, I. E. Day, S. W. Roberts, A. Harpin, J. Drake, and M. Asghari, "Optical dispersion, two-photon absorption and self-phase modulation in silicon waveguides at 1.5 $\mu \mathrm{m}$ wavelength," Appl. Phys. Lett. 80, 416-418 (2002).

[17] E. G. Gamaly, Femtosecond Laser-Matter Interaction: Theory, Experiments and Applications (Pan Stanford Publishing, Singapore, 2011).

[18] S. Bhagavat, and I. Kao, "Theoretical analysis on the effects of crystal anisotropy on wiresawing process and application to wafer slicing," Int. J. Mach. Tool. Manu. 46, 531-541 (2006).

[19] R. R. Gattass, and E. Mazur, "Femtosecond laser micromachining in transparent materials," Nat. Photonics 2, 219-225 (2008). 